\title{
An Evaluation of the Impacts of Authority Coordinating Mechanism on Innovation Ability in Innovation Cluster
}

\author{
Jie Zhen \\ Business School, East China University of Political Science and Law, Shanghai, 201620, China \\ jetzhen@163.com
}

\begin{abstract}
Keywords: Authority coordination, Inter-enterprise relationships, Knowledge sharing, Cooperative innovation capability, Cooperative innovation performance.
\end{abstract}

\begin{abstract}
On the background of the inter-enterprise knowledge sharing, this paper elucidates the connotation and characters of the nonbusiness authority coordinating mechanism and the dimentions of cooperative innovation capability. Then the paper reveals the effect mechanism of the authority coordinating mechanism affecting the cooperative innovation performance with cooperative innovation capability as medium. Also, the paper designs the measurement scale and a formal questionnaire proper and pertinently collects the data. Finally, applying the statistical software, the research has a positive analysis for the constructed theoretical models and assumptions.
\end{abstract}

\section{Introduction}

In innovation cluster, trust, reputation, culture and other factors of relational contract as important supplements to contract coordination mechanism affect the cooperation efficiency among the enterprises. The role of relational contract does not exclude legislative or judicial enforcement action in the performance of the contract [1]. Moreover, The third party arbitration has the deterrent effect. In innovation cluster, the management offices are significant authority coordination force. Therefore, this article makes the theoretical investigation and empirical analysis on the internal mechanism of authority coordinating mechanism.

\section{The Relevant Conception}

(1) Cooperative Innovation Performance. Combined with the research background of collaborative innovation, this article defines cooperative innovation performance as four aspects: the quantity of patents, the quantity of new products, awarded achievements and innovation efficiency.

(2) Cooperative Innovation Capacity. The cooperative innovation ability in this paper is mainly embodied in knowledge sharing and knowledge spillover, which can be expanded specifically in three dimensions of knowledge production ability, knowledge transfer ability and knowledge utilization ability.

\section{Research Hypotheses}

(1) Legitimacy, Cooperative Innovation Ability and Cluster Innovation Performance. The authority based on legitimacy can make or provide public platform for the cooperation among enterprises. Meanwhile, the existence of a third party contact may contribute to the formation of cooperative principles [2]. In addition, the authority coordination platform provides a way of information communication among enterprises. So, for the sake of long-term benefits, enterprises will be more willing to send knowledge and spontaneously improve the ability of knowledge sharing. Thus, this study formulates the hypotheses to be tested as follows:

H1a: The greater the legitimacy of authority coordination is, the stronger the ability of knowledge production is. And ultimately it will influence the innovation performance in innovation cluster positively. 
H1b: The greater the legitimacy of authority coordination is, the stronger the ability of knowledge transfer is. And ultimately it will influence the innovation performance in innovation cluster positively.

H1c: The greater the legitimacy of authority coordination is, the stronger the ability of knowledge utilization is. And ultimately it will influence the innovation performance in innovation cluster positively.

(2) Degree of Obedience, Ability of Cooperative Innovation and Innovation Performance in Cluster. Authority is a kind of relationship of command-obedience. This obedience contains a series of different levels of classification from voluntary obedience to mandatory obedience. In innovation cluster, the strength of authority coordination depends on the level of consistency between the value and pursuit. The highly consistent state will enhance the level of obedience of enterprises in their function range and reach the following and obeying effect that enterprises and cluster share the same fate [3]. At this point, it's easier to stimulate inter-enterprise knowledge sharing activities and further improve their ability of knowledge sharing. On these grounds, this study formulates the hypotheses to be tested as follows:

$\mathrm{H} 2 \mathrm{a}$ : The higher the degree of obedience of authority coordination is, the stronger the ability of knowledge production is. And ultimately it will influence the innovation performance in innovation cluster positively.

$\mathrm{H} 2 \mathrm{~b}$ : The higher the degree of obedience of authority coordination is, the stronger the ability of knowledge transfer is. And ultimately it will influence the innovation performance in innovation cluster positively.

H2c: The higher the degree of obedience of authority coordination is, the stronger the ability of knowledge utilization is. And ultimately it will influence the innovation performance in innovation cluster positively.

(3) Influence Threshold, Cooperative Innovation Ability and Innovation Performance in Cluster. The influence of authority is in a certain area which is called influence threshold. In the influence threshold of authority, authority is a closed system. This system constantly exchanges material and information with other systems, in which process the relationship of obedience and identification is established gradually. The wider the influence threshold of authority coordination is and the more enterprises the influence involves, the more affluent the resource like information and knowledge that can be shared in a united platform is. The utilization of information is the main factor to determine the strength of authority [4]. It can not only raise the cooperation opportunities among enterprises, but also enhance the ability of knowledge sharing, especially knowledge transfer. Therefore, this study formulates the hypothesis to be tested as follows:

$\mathrm{H} 3 \mathrm{~b}$ : the wider the threshold of authority coordination is, the stronger the ability of knowledge transfer is. And ultimately it will influence the innovation performance in innovation cluster positively.

\section{Research Method}

This study adopts enterprise questionnaire method to collect data. The research is conducted as the procedures of questionnaire distribution, data collection, data entry, data analysis. The study select "Tongji rim R\&D design service characteristic industry base" as the main object of samples and 118 knowledge-intensive enterprises in this zone compose the main respondents in this research. And finally 281 valid questionnaires are received.

\section{Empirical Analysis}

\section{Validity and Reliability Analysis on Questionnaire Measurement Scale}

With the item analysis, this article will measure the cooperative innovation ability among enterprises in two dimensions, namely the ability of knowledge production and the ability of knowledge transfer. And the two-dimension measurement of cooperative innovation ability passed 
the validity test. By synthesizing the results of item analysis, EFA and CFA, the one-dimension measurement of authority coordination passes the validity test. Item analysis shows that the measured variables are all identifiable. Also, the measurement scale of innovation performance passes the validation. This article sets the critical value of Cronbach $\alpha$ coefficient, the standard of reliability assessment, as 0.7 . From the result of the test, the internal consistency coefficient of each variable designed in the questionnaire is higher than 0.7 . This demonstrates that the questionnaire scale in this study passes the reliability test.

\section{Model Validation}

According to adjusted intermediary variables, two hypotheses are to be verified, namely H1a' (the better the effect of authority coordination is, the stronger the ability of knowledge production is) and H1b' (the better the effect of authority coordination is, the stronger the ability of knowledge transfer is). By inputting the data of authority coordination and innovation ability scale and making SEM analysis with AMOS7.0, we can get the conclusion that H1a' and H1b' are verified.

Here the hypotheses to be verified are $\mathrm{H} 4 \mathrm{a}$ (knowledge production ability has positive relationship with cooperative innovation performance) and $\mathrm{H} 4 \mathrm{~b}$ (knowledge transfer ability has positive relationship with cooperative innovation performance). By inputting the data of cooperative innovation ability and innovation performance scale and making SEM analysis with AMOS7.0, H4a and $\mathrm{H} 4 \mathrm{~b}$ are verified.

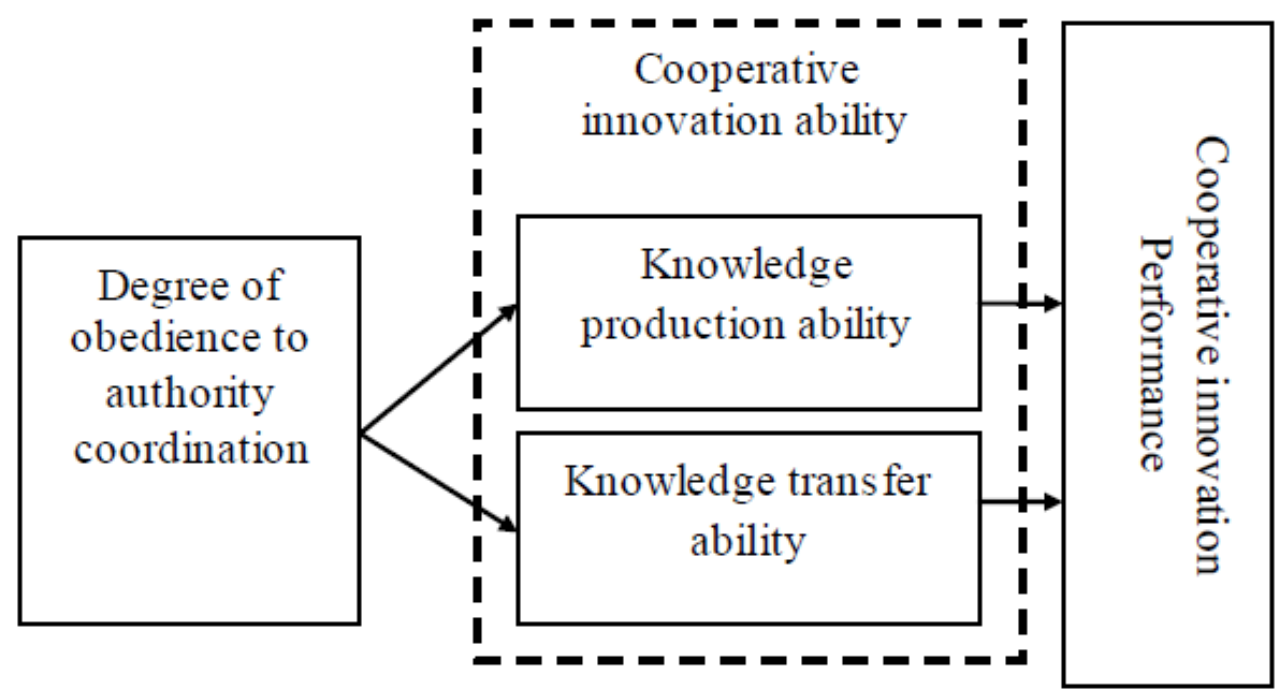

Fig.1 The Relational Structural Model Intended To Be Verified

Through the verification and adjustment of each latent variable measurement model as well as the analysis and verification to each submodel, this article probes into the full-model structural relationship of all latent variables. The full-model structural relationship model to be verified is shown in figure1. When inputting the data of all-latent-variable measurement scale and making SEM analysis with AMOS7.0, overall, the verification of relationship hypothesis of all-latentvariable model analysis is in accordance with that of each submodel.

\section{Conclusion}

Organizations as management departments in innovation cluster guide and coordinate the interfirm cooperative innovation conduct in cluster on the basis of their advantages of knowledge, information, policies etc. By improving the cooperative innovation ability among enterprises through knowledge production ability and knowledge transfer ability, the organizations will ultimately exert positive influence on cooperative innovation performance. The authority coordination in innovation cluster can be understood from the features of three factors, legitimacy, degree of obedience and influence threshold. But in the process of enhancing cooperative innovation ability and influencing innovation performance, authority coordination presents one- 
dimension. On the one hand, it illustrates that in cluster the enterprises' evaluation to authority coordination tend to be a general impression, on the other hand, it demonstrates that the authority coordination organizations in cluster should improve their images from the above three aspects to give full play to the regulating effect of authority coordinating mechanism on the enhancement of innovation cluster performance.

\section{Acknowledgement}

The research work was supported by Chinese National Social Science Foundation under Grant No. 12CJL012 and Chinese National Natural Science Foundation under Grant No. 71272048 and 2015 Key Project of Shanghai Soft Science Research Plan in the Action Plan of Science and Technology Innovation under Grant No. 15692101800 and 2015 Shanghai Social Sciences Planning Project under Grant No. 2015JG009-BGL307 and 2014 Research Project on Teaching Reform and Development of East China University of Political Science and Law.

\section{References}

[1] Macaulay, S., Non-contractual Relation in Business: A Preliminary Study. American Sociological Review, 28(1), pp. 55-67, 1963.

[2] Van den Berg L. Braun E. \& Van Winden W., Growth Clusters in European Cities: An Integral Approach. Urban Studies. 38 (1), pp. 186- 206, 2001.

[3] Liping Wang. \& Yan Zhou. Authority Mechanism in the Process of Management. Journal of Renmin University of China, 4, pp. 66-70, 2007.

[4] Munson C L., Rosenblatt M.J. \& Rosenblatt Z., The Use and Abuse of Power in Supply Chain, TEEE Engineering Management Review, Second Quarter, pp. 81-91,2000. 